\title{
Cultura y procesos cognitivos: hacia una psicología cultural
}

\author{
MANUEL L. DE LA MATA \\ JUAN DANIEL RAMÍREZ \\ Universidad de Sevilla
}

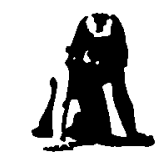

Resumen

Se bace una revisión de las investigaciones cognitivas transculturales de las últimas décadas. De estos estudios se deriva una serie de problemas metodológicos que cuestionan la validez de sus resultados. En el plano teórico, por otra parte, ha prevalecido un enfoque de caja negra que no ba permitido desvelar los mecanismos de influencia de lo cultural sobre lo cognitivo. Se defiende un planteamiento nuevo, que se sitúa en la linea del enfoque sociocultural de la psicología soviética, que centra los análisis en los contextos culturales y que toma las nociones de actividad y zona de desarrollo próximo como herramientas conceptuales para el estudio de la relación entre cultura y procesos cognitivos.

Palabras claves: Cultura, procesos cognitivos, psicología transcultural, contextos culturales, actividad, zona de desarrollo próximo.

\section{Culture and cognitive processes. Towards a cultural psychology}

\section{Abstract}

In this paper a review of cross-cultural cognitive research in the last decades is carried out. From these studies a number of methodological problems questionning the validity of their results is derived. From a theoretical point of view a black-box approach has dominated the field. This approach is not useful for specifying the mechanisms relating to culture or cognition. A new approach, based in a Soviet sociocultural framework is supported. It focuses on the analysis of cultural contexts and take the notions of activity and zone of proximal development as conceptual tools for studying the relation between culture and cognition.

Keywords: Cognitive processes, cross-cultural psychology, cultural contexts, activity, zone of proximal development.

Dirección del autor: Universidad de Sevilla, Laboratorio de Actividad Humana, Apartado 3128 , Sevilla.

Original recibido: Febrero 1988. Revisión recibida: Octubre 1988. Aceptado: Octubre 1988. 
Después de algunas décadas de investigación transcultural y en un momento de influencia creciente de la perspectiva sociocultural en la psicología occidental, la pregunta que cabe hacerse es la siguiente: ¿hay indicios que apunten hacia el desarrollo de una psicología de la cultura? Si la respuesta a la pregunta es positiva, la psicología podría verse liberada de la oposición no resuelta entre individuo y sociedad. Esta dicotomía hace irreductibles e incomunicables los dos polos en torno a los cuales se dividen de forma maniquea las ciencias sociales. Sin embargo, con mejor o peor fortuna, la psicología transcultural ha logrado hacernos reflexionar sobre los aspectos universales y diferenciales de la conducta humana. Una reflexión sobre las aportaciones de la psicología transcultural es indispensable en este momento, en el que la psicología tiene dificultades para entender el modo en que afectan cognitivamente al individuo los grandes cambios culturales que han venido operándose a lo largo de nuestro siglo y que continúan operándose en el presente. La reflexión sobre el papel de la cultura es fundamental para superar ciertas limitaciones que la psicología viene arrastrando. La dicotomía, con tintes de irreductible, entre individuo y sociedad es una de esas limitaciones heredadas del siglo XIX.

\section{DIFERENCIAS COGNITIVAS ASOCIADAS A FACTORES CULTURALES}

La psicología transcultural ha señalado la existencia de diferencias entre individuos de cultura occidental y sujetos que viven en condiciones culturales diferentes a las que caracterizan a las sociedades del mundo desarrollado (educación formal, urbanización, «modernidad», etc.). Estas diferencias se ponen de manifiesto en diversos procesos cognitivos. A continuación pasaremos revista a algunas de ellas.

\section{Percepción}

La investigación transcultural ha afirmado la presencia de variantes en la conducta perceptiva ligadas a la cultura. En primer lugar, hay diferencias entre los sujetos de cultura occidental y los de otras culturas en el manejo de los estímulos bidimensionales y en la interpretación de las claves de profundidad de dichos estímulos (Cole y Scribner, 1974; Rogoff, 1981). Los sujetos no occidentales (en sus formas de vida) interpretan con mayor dificultad las claves de profundidad de los estímulos bidimensionales.

Respecto a la percepción de los colores, Luria (1979) encontró diferencias asocidas a las condiciones culturales en la denominación y la agrupación de sus tonalidades. Los sujetos de Uzbequistán (Asia central soviética) que vivían en condiciones culturales "tradicionales" a menudo denominaban los colores por el nombre de los objetos que tenían dichos colores. Aunque conocían y empleaban también nombres categoriales para los colores, la frecuencia de empleo de estos nombres era mucho menor que la que se encontraba en los individuos de condiciones de vida "modernas" (trabajadores de granjas colectivas, sujetos escolarizados...). Igualmente agrupaban las tonalidades por luminosidad o saturación más que por el criterio de pertenencia al mismo color general. 
En suma, estos datos sobre diferencias culturales en la percepción parecen mostrar que ésta tiene un carácter más particular y menos conceptual en los individuos no "occidentalizados" respecto a los de cultura occidental (Bruner, Greenfield y Olver, 1965; Cole y Scribner, 1974; Luria, 1979) y una preferencia selectiva por las propiedades externas de los estímulos (como el color), que no va siendo sustituida con la edad por atributos como número y función, tal como ocurre en los sujetos de cultura occidental (Bruner, Greenfield y Olver, 1965; Cole y Scribner, 1974; Luria, 1979).

\section{Agrupación y categorización}

Uno de los temas más estudiados, si no el que más, en la psicología transcultural ha sido el modo en que agrupan o categorizan los estímulos los individuos de otras culturas.

En su expedición a Uzbequistán de los años treinta, Luria (1979) llevó a cabo una serie de investigaciones sobre este tema. Concluyó de ellas que aquellos individuos que se encontraban inmersos en condiciones de vida tradicionales tendían a agrupar los estímulos sobre la base de su pertenencia a situaciones prácticas comunes en las que aparecían los estímulos. Por ejemplo, al proponérseles la terna de objetos "pala", "leño" y "hacha" para que agrupasen dos de ellos, excluyendo un tercero, tendían a agrupar «hacha» y «leño", dado que "el hacha sirve para cortar el leño", rechazando la pareja "pala-hacha», cuya base es la categoría «instrumentos de trabajo». Esta tendencia fue observada por Luria tanto en tareas de emparejamiento como la descrita cuanto en otras de agrupación de varios elementos, en las que rechazaban la realización de agrupaciones categoriales taxonómicas. En contraste, los sujetos cuyas condiciones de vida podían calificarse de modernas mostraron una clara preferencia por el empleo de criterios categoriales taxonómicos en las agrupaciones.

Las tendencias apuntadas por Luria parecían confirmarse en una serie de investigaciones en las que se observaba que los sujetos no escolarizados de otras culturas tendían a efectuar sus agrupaciones basándose en atributos externos de los objetos, tales como el color (Bruner, Greenfield y Olver, 1965; Cole y Scribner, 1974; Greenfield, 1974; Scribner y Cole, 1981; Serpell, 1969; Sharp, Cole y Lave, 1979). Los sujetos escolarizados de otras culturas mostraban, en cambio, tendencias muy semejantes en sus respuestas a las de los niños occidentales.

Recientemente hemos realizado, junto a otros compañeros (Ramírez et al., 1986), una investigación con adultos andaluces, en la que los resultados han sido bastante coincidentes con los de Luria y otros autores. La tarea empleada en este trabajo ha sido de emparejamiento de dos elementos de una terna, rechazando al mismo tiempo un tercero. Tras inducir a los sujetos a que emplearan el concepto de vida como base de los emparejamientos, se les pedía que eligieran un dibujo, de entre dos que aparecían en la parte derecha de la lámina, para emparejarlo con otro, colocado éste en la parte izquierda de la misma lámina, y que representaba siempre a un ser vivo, animal o planta. La tarea estaba graduada en niveles de dificultad creciente, de modo que la relación entre los seres vivos de la terna era más compleja, más «lejana» en términos de tipicidad y grado de inclusión en la clase (Rosch, 1975; Soto, 1981). Así, por ejemplo, mientras que en la pri- 
mera lámina los dos elementos vivos eran «caballo» y «cerdo» (dos mamíferos, es decir, dos animales prototípicos), en la última eran «eucalipto" y "gamba" (el segundo de ellos no era una planta, sino un animal, atípico además). El aumento de la dificultad, de la "distancia" entre los elementos a emparejar se tradujo en un incremento del número de emparejamientos de tipo asociativo o "contextual", es decir, basados en relaciones de pertenencia a situaciones prácticas comunes. Un ejemplo de este tipo de respuestas sería la de aquellos que consideraron que el «eucalipto» debía emparejarse con la «gamba» porque «se pueden comer gambas a la sombra de un eucalipto". Este tipo de respuestas fue predominante en todos los sujetos. Aunque algunos de ellos habían estado acudiendo al centro de adultos durante unos tres años (si bien de forma poco constante en la mayoría de los casos), esa experiencia escolar no había modificado las tendencias que halló Luria y que han hallado igualmente otros autores en los sujetos de cultura tradicional.

La importancia de los rasgos externos en la clasificación ha sido también resaltada por los antropólogos en el terreno de la clasificación de los reinos animal y vegetal (Berlin, 1972, 1978). En este sentido hay que decir que las investigaciones más recientes tienden a enfatizar el peso de la semajanza perceptiva como aspecto configurador de las clases, en lugar de la significación funcional de las plantas y de los animales en el plano de la supervivencia.

En resumen, de las investigaciones citadas sobre agrupación y categorización parece deducirse que los sujetos de culturas tradicionales tienden a agrupar los estímulos sobre la base de relaciones dadas en el contexto de actividad práctica y que cuando categorizan utilizando un criterio taxonómico, éste suele estar relacionado con los atributos más externos, menos abstractos de los estímulos.

\section{Memoria}

Las investigaciones sobre diferencias culturales en la memoria se han centrado sobre todo en tareas de recuerdo libre de estímulos agrupables, tareas de memorización central e incidental y de aprendizaje de pares asociados.

En el primer tipo de tareas se ha medido, por lo general, tanto el número de elementos recordado como la agrupación en la codificación y en el recuerdo. Lo que la mayoría de los autores ha encontrado es una relación positiva entre la presencia de ciertas condiciones culturales como la escolarización y otras y el número de elementos recordado, así como la agrupación en la codificación y en el recuerdo (especialmente la formación de categorías taxonómicas) (Brown, 1977; Cole y Scribner, 1974, 1977; Rogoff, 1981; Sharp, Cole y Lave, 1979; Scribner y Cole, 1981; Wagner, 1981; Zivian y Darjes, 1983).

Respecto a las tareas de aprendizaje central e incidental, parece coincidente la evidencia de que los sujetos occidentales tienden a recordar mayor cantidad de información central e incidental. Es interesante hacer notar, igualmente, que el recuerdo de los individuos escolarizados occidentales es más selectivo que el de los de experiencia cultural tradicional. Esto implica que en términos relativos los primeros recuerdan mayor cantidad de ma- 
terial central (en relación a la cantidad total de información recordada) que los segundos en los que la diferencia entre un tipo y otro de material recordado es menor (Wilkinson et al., 1979; Rogoff, 1981).

En tareas de aprendizaje de pares asociados hay también superioridad de los resultados que obtienen por lo general los individuos de cultura occidental sobre los sujetos no escolarizados de otras culturas. Esta superioridad se manifiesta con más vigor cuando los pares de lista están poco relacionados (Sharp, Cole y Lave, 1979).

\section{Razonamiento y resolución de problemas}

El tema del razonamiento y la resolución de problemas no ha recibido mucha atención en la psicología transcultural. No obstante, existen algunas investigaciones que podemos citar. El tipo de situaciones de estudio empleadas con más frecuencia ha sido la presentación de silogismos lógicos, en los que se dan dos premisas al sujeto, verbalmente formuladas, debiendo éste combinarlas para deducir una conclusión igualmente verbal.

Utilizando este tipo de tareas, Luria (1979) llegó a la conclusión de que los individuos cuyas condiciones de vida hemos calificado de tradicionales se negaban a realizar la resolución de los problemas dentro de los límites de éstos, del modo en que eran formulados por el investigador. Para Luria, la resolución de problemas exige "que tal resolución transcurra del marco de un sistema lógico cerrado. Hablando de otro modo, las personas que resuelven un problema no pueden salir del sistema lógico de relaciones que están limitadas por los datos que han sido formulados como condiciones del problema" (Luria, 1979, p. 152). Para resolver estas tareas, los sujetos de Luria solían hacer uso de su experiencia personal reemplazando la resolución lógica discursiva por el análisis de los datos concretos de la experiencia personal. Así, cuando se les proponía un silogismo del tipo:

*Los osos de los países muy fríos son blancos.

Nueva Zembla es un país muy frío.

¿Cómo son los osos de Nueva Zembla?»

respondían por lo general que ellos no habían estada nunca en Nueva Zembla y, por tanto, no podían saber cómo son allí los osos. Prevalecía, al decir de Luria, la referencia a la experiencia práctica sobre las relaciones lógico-verbales que fundamentan la deducción. Parecidas respuestas a las halladas por Luria se han encontrado en otras investigaciones transculturales que han usado problemas lógicos semejantes a los descritos (Cole, Gay y Glick, 1971; Cole y Scribner, 1974; Scribner y Cole, 1981; Sharp, Cole y Lave, 1979). En general, eran los sujetos más «occidentalizados" (escolarizados, urbanos, etc.) los que resolvían los silogismos ateniéndose a los límites establecidos por las premisas y sin salirse de ellos.

\section{INSUFICIENCIAS Y LIMITACIONES DE LA INVESTIGACION TRANSCULTURAL}

Los datos presentados en el apartado anterior, procedentes de la investigación transcultural, podrían hacernos pensar que existe una clara diferencia entre los procesos cognitivos de los sujetos de otras culturas y los 
de los sujetos de cultura occidental moderna. Desde este punto de vista, el pensamiento del individuo de cultura tradicional se caracterizará por una fuerte dependencia del contexto, de los estímulos que configuran las situaciones prácticas y una cierta incapacidad para trascender los límites de dichas situaciones. Esto abundaría en la hipótesis de que los procesos cognitivos son diferentes en diferentes culturas y dependen de variables culturales (ver Pick, 1980, para una exposición más detallada de la hipótesis).

Sin embargo, no es esa la visión que predomina actualmente en los investigadores ni, desde luego, la que defendemos en este trabajo. Deliberadamente hemos omitido una gran cantidad de estudios que matizan las ideas y los resultados expuestos hasta el punto de hacernos modificar las conclusiones generales que la investigación transcultural en las diferentes áreas del funcionamiento cognitivo suscita acerca de las diferencias asociadas a la cultura y, en sentido más general, acerca de las relaciones entre cultura y conocimiento.

Comenzando por la percepción, podemos citar un trabajo de Cole, Gay y Glick (1968), en el que se comparaban destrezas en la discriminación perceptiva de universitarios norteamericanos y de sujetos adultos kpelle de Liberia. Cuando se proyectaba un conjunto de puntos en la pantalla de un taquistoscopio, los universitarios norteamericanos discriminaban mejor el número que los adultos kpelle. Sin embargo, si en lugar de un conjunto de puntos eran unas piedras dispuestas en un montón lo que se les presentaba para que determinaran su número, los resultados cambiaban radicalmente: eran ahora los adultos kpelle los que mostraban mayor destreza. Aunque los universitarios norteamericanos mejoraban la discriminación con el ejercicio, no lograban alcanzar la eficacia de los liberianos. Se observa, pues, que los resultados obtenidos estaban muy relacionados con los materiales empleados en la investigación.

La importancia de los materiales queda patente también, y de modo especial, en investigaciones sobre clasificación y formación de conceptos. Ya Price-Williams (1962), en un estudio realizado en Nigeria, observó que el empleo de objetos tridimensionales familiares (animales y plantas) en lugar de estímulos de dos dimensiones hacía que los resultados alcanzados por los niños analfabetos fuesen semejantes a los de aquellos que habían asistido a la escuela. El análisis de las respuestas verbales y de las justificaciones dadas por los sujetos tras realizar las clasificaciones puso de manifiesto un hecho interesante adicional. Las justificaciones relativas a la forma en que habían clasificado los animales tendieron a ser de índole concreta. En cambio, cuando justificaron las agrupaciones de plantas recurrieron en su mayoría a un rasgo tan abstracto como el carácter comestible o no del vegetal. Esto nos hace ver que el carácter abstracto o concreto de la respuesta dependía también de la procedencia de los objetos, no sólo del grado de familiaridad de los materiales y de su forma de presentación.

Podemos citar muchas otras investigaciones que demuestran la influencia de la familiaridad con los estímulos sobre los resultados de clasificación. Irwin y McLaughlin (1970) realizaron dos experimentos en los que los sujetos eran niños escolarizados y adultos analfabetos cultivadores de arroz en Liberia central. En el primer experimento los niños escolarizados fueron superiores en destreza a la hora de clasificar tarjetas con figuras geométricas que variaban según tres dimensiones (color, forma y número). Sin 
embargo, cuando lo que los sujetos debía clasificar no eran figuras geométricas sino tazones de arroz (y el arroz difería igualmente en tres dimensiones: con o sin cascarilla, tamaño y tipo), los resultados se invirtieron por completo. Los más hábiles fueron ahora los adultos analfabetos. Resultados semejantes aparecen en los trabajos de Deregowski y Serpell (1971), Fjellman (1971) e Irwin, Schafer y Feiden (1974) empleando materiales del mismo tipo.

En cuanto a la investigación sobre memoria hay que decir que el panorama descrito en el apartado anterior varía cuando se examinan estudios que han empleado tareas diferentes a las anteriores o han introducido variantes en las situaciones de prueba. Veamos algunos ejemplos.

En un trabajo sobre memoria de reconocimiento, Cole et al. (1971) no encontraron inferioridad de los sujetos no escolarizados frente a los escolarizados. Wagner (1978) tampoco halló diferencias asociadas a la escolarización al estudiar el reconocimiento de fotografías de alfombras orientales con sujetos de diferente edad, con y sin experiencia escolar, procedentes de Marruecos y EE.UU. Es interesante resaltar que la ejecución superior en esta prueba fue obtenida por un grupo de vendedores de alfombras, lo que destaca la importancia de la familiaridad con los materiales en la determinación de la memoria, aun en el caso de la memoria de reconocimiento, supuestamente menos influida por factores de estrategia. Con otras tareas de memoria visual algo diferentes (reconstrucción de escenas), los resultados han vuelto a mostrar que no había diferencias entre grupos de individuos escolarizados y no escolarizados (Rogoff y Wadell, 1982).

Incluso en tareas de recuerdo libre es posible también encontrar ejemplos en que el rendimiento ofrecido por sujetos con escasa o ninguna experiencia escolar era semejante al que obtenían los individuos occidentales que habían acudido a la escuela. El empleo de una clave externa concreta unido a instruccciones que inducían a recordar todos los elementos de una categoría antes de pasar a la siguiente mejoraba el recuerdo de los sujetos kpelle no escolarizados hasta igualarlo al de individuos escolarizados occidentales (Cole el al., 1971). Por otra parte, Scribner (1974) demostró que el nivel de agrupación en el recuerdo era semejante en un grupo de trabajadores del monte analfabetos del grupo kpelle y en otro de estudiantes universitarios del mismo pueblo liberiano, si bien era diferente la naturaleza de las agrupaciones ( predominantemente asociativas en los primeros y de tipo categorial taxonómico en los estudiantes).

Por último, las investigaciones que han empleado historias como material no han encontrado generalmente diferencias en el recuerdo entre individuos escolarizados y/o de cultura occidental e individuos no escolarizados y/o de cultura tradicional (Cole y Scribner, 1974; Dube, 1982; Mandler et al., 1980) o, cuando ha habido diferencias, éstas han sido de índole muy específica (Deregowski, 1970).

En general, puede decirse que las diferencias en la memoria asociadas a factores culturales aparecen sobre todo en tareas en las que el material no está estructurado suficientemente y es el sujeto quien debe imponerle una organización, una estructura (listas de palabras, dibujos, etc.). Fuera de la escuela no parece haber muchas situaciones en que el individuo deba operar de este modo. Cuando, por el contrario, la tarea o el material poseen una organización propia y el individuo puede hacer uso de las relaciones 
significativas entre los estímulos, como ocurre habitualmente en la vida cotidiana, las diferencias culturales desaparecen (Cole y Scribner, 1974, 1977; Paris et al., 1985). Como afirman Rogoff y Mistry (1985), los individuos no occidentales carentes de experiencia escolar tienen, sin embargo, la misma experiencia que los occidentales en recordar información inserta en un contexto estructurado, en usar estrategias que incorporan la información existente usando las relaciones significativas entre los elementos como ayuda para el recuerdo. En tales casos, las diferencias culturales se reducen o desaparecen por completo.

También en la investigación sobre resolución de problemas aparecen dificultades que nos obligan a cuestionar la validez de las inferencias sobre los procesos de deducción y razonamiento de los individuos de otras condiciones culturales. Cole y Scribner (1974) señalan que las respuesta a los problemas de lógica verbal no permiten extraer conclusiones acerca de los procesos lógicos de los sujetos estudiados, ya que existirían factores previos de comprensión de la información y de la tarea. Como apoyo a esta idea citan un trabajo de Gay (1971) en el que se utilizó un material sacado del folklore de los kpelle de Liberia. Al comparar los resultados de sujetos kpelle escolarizados y analfabetos y estudiantes universitarios norteamericanos observó que todos obtuvieron el mismo tanto por ciento de respuestas erróneas. Sin embargo, eran los universitarios estadounidenses los que alcanzaban un mayor número de respuestas correctas. Un análisis más fino de los resultados reveló que la diferencia estribaba en que cuando se proponía un problema de dos alternativas en el que las dos eran desagradables, si bien una era peor que la otra, los universitarios norteamericanos tendían a elegir la menos mala de las opciones, mientras que los sujetos kpelle se desligaban del asunto generalmente, no aceptaban elegir entre dos posibilidades malas. Esto hace pensar que eran factores de índole diferente a la habilidad de deducción los que explicaban las diferencias en la ejecución de unos y otros. En la misma linea de señalar la intervención de factores ajenos a la capacidad lógica, Rogoff (1981) defiende que no hay diferencia en el razonamiento lógico entre los sujetos escolarizados y los no escolarizados, sino en la disponibilidad a aceptar los silogismos del problema. Los individuos no escolarizados se sentirían incómodos respondiendo a un problema cuyas premisas no pueden verificar. Cuando estos sujetos intentan tratar el problema como una unidad lógica, continúa diciendo Rogoff, muestran una capacidad lógica semejante a la de los sujetos escolarizados.

Scribner y Cole (1981) se inclinan a pensar que son factores de naturaleza metalingüística, es decir, de comprensión lingüística del mensaje que transmiten las premisas, los que resultan cruciales en los resultados que los individuos no escolarizados obtienen en tareas de resolución de problemas lógicos verbales. En general, puede decirse que no hay evidencia de que existan diferentes procesos lógicos en unos y otros sujetos ni, por supuesto, parece defendible a estas alturas la idea de que hay una «lógica primitiva" (Levy-Bruhl, cit. en Cole y Scribner, 1974).

No parece que pueda mantenerse, pues, la idea de que los sujetos de cultura occidental son superiores a los de condiciones culturales diferentes en sus procesos cognitivos básicos. Nos inclinamos a pensar, por contra, con la mayoría de los autores actuales, que las diferencias residen más en las situaciones en que estos procesos cognitivos se aplican que en la exis- 
tencia o inexistencia de un proceso en un grupo cultural dado (Cole et al., 1971; Pick, 1980). No obstante, esta idea misma, aunque parece ser la que mejor se adapta a los resultados de muchos estudios, tampoco se deriva directamente de los datos. En realidad, los últimos años de investigación transcultural han puesto de manifiesto sobre todo un conjunto de problemas sin resolver que cuestionan la validez de toda la investigación transcultural $e$, incluso, de gran parte de la investigación cognitiva en general.

Rogoff (1981), a propósito de la influencia de la escolarización, ha sistematizado estos problemas y plantea la existencia de diferentes tipos de sesgo que afectan a la validez y el alcance de los datos obtenidos. Veamos cuáles son estos sesgos:

Sesgos de selección de los sujetos. En muchos estudios no se ha controlado el hecho de que los sujetos escolarizados puedan diferenciarse de los no escolarizados en factores como estatus socioeconómico, educación de los padres, expectativas propias y de familia acerca de lo que significa la escuela y el aprendizaje escolar, etc., que pueden afectar a los resultados obtenidos en pruebas cognitivas. De hecho, podemos citar aquí un trabajo posterior de Stevenson (1982) en el que se comprobaba la existencia de diferencias cognitivas previas a la asistencia a la escuela entre los niños que comienzan a acudir a ella y los niños que no lo hacen. Factores ambientales y de entorno familiar, sobre todo, eran los que explicaban estas diferencias previas a la escolarización misma.

Sesgos relacionados con las propias pruebas. Se nos plantea aquí la cuestión de la familiaridad con la prueba. Esta familiaridad, a su vez, puede desglosarse en tres componentes, tres dimensiones separadas de familiaridad que evidentemente se relacionan con los resultados obtenidos en las pruebas:

a) Familiaridad con los materiales. Ya hemos visto que afecta a los resultados. El empleo de materiales familiares para los sujetos escolarizados y poco conocidos por los no escolarizados (como figuras geométricas, por ejemplo) puede ser el responsable de los bajos resultados que estos sujetos obtienen en muchas pruebas, más que una auténtica falta de capacidad o destreza cognitiva. A propósito de la clasificación, Greenfield (1974) aporta unà interesante distinción entre diferentes dimensiones de familiaridad que tienen que ver con los agrupamientos realizados: familiaridad con el modo de representación (bidimensional o en tres dimensiones, por ejemplo); familiaridad con los rasgos relevantes del objeto (por ejemplo, el azul, el triángulo, etc.); familiaridad con una dimensión (color, forma, etc.) y familiaridad con una relación objeto-dimensión (es decir, si los individuos de un grupo determinado categorizan los objetos particulares de acuerdo con la dimensión usada). Para que una tarea sea culturalmente apropiada en cuanto a materiales deberá tener en cuenta estos criterios.

b) Familiaridad con el lenguaje. En muchas de las investigaciones realizadas el lenguaje empleado en las pruebas ha sido más familiar para los individuos escolarizados que para los no escolarizados.

c) Familiaridad con la situación y con las demandas de la prueba. Una diferencia obvia entre los sujetos escolarizados y los no escolarizados es la familiaridad con el hecho de ser puestos a prueba, lo cual podría dar cuenta de muchos de los resultados obtenidos. Como señala Goodnow (1976), 
las diferencias entre los grupos escolarizados y no escolarizados pueden deberse en gran medida a la interpretación del problema que se está resolviendo y a diferencias respecto a los "métodos" de resolución considerados más aceptables (rapidez, alcanzar la solución con un número mínimo de pasos o de redundancia, solución original versus deferencia hacia la autoridad, etc.). Igualmente, los sujetos escolarizados están más familiarizados con situaciones de entrevista o prueba en las que un adulto de estatus superior, que ya conoce la respuesta a la cuestión, requiere información de una persona de estatus inferior (como un niño, por ejemplo). La escolarización ofrece también práctica con ciertas convenciones como las que se emplean para representar la profundidad en dibujos de dimensiones, el género de problemas en los que debe tomarse en cuenta sólo la información dada en el problema para alcanzar la solución y el formato común de los elementos de la prueba. La escolarización puede dar también una visión de lo que es «inteligente» (por ejemplo, la agrupación por categorías taxonómicas más que por pertenencia a contextos prácticos comunes).

Otros aspectos de las pruebas que pueden ser familiares solamente para los sujetos con experiencia en la escuela, como el énfasis en la rapidez de la ejecución, el trabajar independientemente más que colaborando, etc.

Pero el rasgo de las situaciones de prueba que resulta más novedoso para los sujetos no escolarizados tiene que ver con el objetivo de la actividad. Efectivamente, en otras culturas y, en la nuestra, en grupos cuyas condiciones de vida podemos calificar de tradicionales las destrezas cognitivas se ponen de manifiesto en situaciones prácticas que definen por sí mismas el objetivo de la actividad. Así, por ejemplo, un individuo debe comprar un determinado conjunto de objetos, para lo cual deberá recordar esos objetos. En este caso la acción de memorizar está inserta en una actividad más general que hace necesaria dicha acción y define su objetivo (el recuerdo de los objetos para comprarlos). Este tipo de situaciones es también propio de nuestra cultura, por supuesto. Pero lo que no es tan frecuente en otros grupos culturales es la existencia de situaciones que, aunque tengan referente real, ese referente no está presente en momento de realizar la actividad, por lo que el manejo de la realidad es puramente simbólico. Es decir, son situaciones en que se trabaja con información descontextualizada. En estas situaciones se exige al sujeto, por ejemplo, que recuerde una gran cantidad de información. Para ello debe emplear unos medios instrumentales (extracción de las ideas más significativas y su ordenación, el repaso, etc.) sin que en este caso el objetivo venga definido previamente en el curso de una actividad práctica en la que tenga sentido la memorización. Tiene que ser ahora el individuo el que establezca el objetivo (en función de una orden externa, en función de otro objetivo remoto, etc.) y elija y ponga en marcha los medios necesarios para su consecución. Y todo ello debe hacerlo con información sacada de contexto y sin el soporte significativo que proporciona la realización de una actividad práctica en el contexto cotidiano, en el curso de la cual debe ejercitarse la destreza cognitiva. Este tipo de situaciones, que son las escolares ( $y$ muchas otras de las sociedades tecnológicas), son también las de la mayoría de las pruebas cognitivas empleadas en la investigación transcultural. Es comprensible que los sujetos escolarizados obtengan mejores resultados en ellas que los que no han ido a la escuela. 


\section{3. ¿EXISTEN DIFERENCIAS?}

A la vista de lo expuesto en el apartado anterior, la pregunta a hacerse es: ¿existen realmente diferencias cognitivas entre los sujetos de diferentes condiciones culturales? Para responder a esta pregunta habría que volver a las dimensiones de familiaridad anteriores, especialmente el tipo de materiales y situaciones manejados.

Es evidente que la investigación transcultural ha señalado que existen diferencias entre los sujetos de diferentes condiciones culturales. Con materiales muy simbólicos y abstractos (figuras geométricas, estímulos bidimensionales, etc.) los sujetos escolarizados occidentales suelen obtener mejores resultados en pruebas de categorización, memoria, etc. Se trata del tipo de estímulos cuyo manejo está muy ligado a la escuela y, en general, a las sociedades tecnológicas, en las que los individuos han de conocer sus convenciones para adaptarse a ellas.

Igualmente se observa que existen diferencias entre individuos de distintas culturas en el tipo de situaciones en que estos individuos realizan sus destrezas. Ya hemos visto que situaciones como las de laboratorio, en las que las destrezas cognitivas deben ejercitarse fuera del contexto de là actividad práctica, dificultan mucho la ejecución de los individuos analfabetos o de culturas no literarias. En las situaciones cotidianas de otras culturas, y en muchas de nuestra cultura, el empleo de las destrezas cognitivas viene dado y ocurre en el curso de las actividades prácticas social e individualmente significativas. No es que el individuo se proponga "poner a prueba» sus destrezas cognitivas, sino resolver de modo adaptado las exigencias de la actividad que realiza. Por el contrario, cuando esta exigencia cognitiva no viene inserta en el curso de la actividad práctica significativa, siendo el objetivo "el ejercicio cognitivo en sí mismo" y/o la destreza cognitiva es necesaria para resolver un problema muy descontextualizado, con estímulos muy abstractos y en el que el objetivo de la actividad (por el que debe ponerse en juego la destreza) no está marcado por el contexto práctico, los individuos que no han asistido a la escuela parecen estar en situación poco ventajosa frente a aquéllos cuya vida transcurre en las llamadas sociedades tecnológicas y han asistido a la escuela.

En otro orden de cosas parece que existe un cierto acuerdo acerca del hecho de que un rasgo frecuente en sujetos de culturas no literarias es la falta de verbalizaciones de los atributos que determinan la inclusión de unos determinados elementos en una clase. La educación informal no fomentaría en la misma medida que la educación formal el análisis, por la vía del lenguaje, de los rasgos y propiedades más señeras de los objetos. En realidad, hablar sobre los rasgos y atributos de la tarea es sólo un ejercicio cognitivo más, algo que no es necesario para el desarrollo de las actividades altamente contextualizadas, predominantes en la vida de estos individuos.

Podemos relacionar con ello el hecho de que las investigaciones sobre la memoria en contextos culturales distintos señalan que las diferencias entre unos sujetos y otros se dan, no en las destrezas de memoria básicas ni con materiales compartidos por unas culturas y otras (como sería el caso de las historias) (Mandler et al., 1980), sino a la hora de poner en juego destrezas de control de naturaleza estratégica muy finas, con materiales abstractos y en ámbitos muy descontextualizados y alejados de la experiencia 
cotidiana con objetos. Parece que situaciones como éstas es difícil que se encuentren fuera de la escuela, y, por tanto, los que han asistido a ella se desenvuelven mejor que los que no lo han hecho (Brown, 1977; Cole y Scribner, 1977). Hay otro hecho digno de considerar aquí también, que es la mayor facilidad de los sujetos escolarizados a la hora de explicar verbalmente' las actividades realizadas, lo que a su vez podría facilitar el control de esas actividades.

Cambiando de campo de investigación, hay que decir que la mayoría de los estudios transculturales sobre resolución de problemas ponen de manifiesto que las diferencias entre sujetos de ésta y otras culturas estribaban, no en los procesos lógicos de base, sino en otros factores como la disposición a aceptar los silogismos del problema (Rogoff, 1981). Para Scribner (1977) los silogismos verbales representarían un género de lenguaje especializado, diferente de otros. Sería la práctica con este género de lenguaje lo que permite al sujeto llegar a manejar versiones más complejas de dicho lenguaje. Tal práctica sería frecuente en la escolarización occidental. Olson (1977), por su parte, hace hincapié en que lo que está en juego en un problema de tipo considerado es si se asume o no que el significado está completamente explícito y autónomo en el problema. En esta misma línea de interpretación, Scribner y Cole (1981) trataron de determinar los aspectos metalingüísticos implicados en la resolución de problemas de lógica verbal en sujetos vai de Liberia. Cuando el sujeto participaba en una conversación sobre gramática y significado de las palabras en su propia lengua, la resolución de dichos problemas mejoraba. Esta conversación favorecía la focalización sobre el problema silogístico mismo y sobre el contenido de sus premisas, rompiendo toda dependencia de sus experiencias previas y de sus sistemas de creencias.

Puede decirse, pues, que las diferencias entre individuos de condiciones culturales diversas, de modo especial entre aquellos que han asistido a la escuela y los que no lo han hecho, estriban en la facilidad con que se desenvuelven unos y otros con materiales y situaciones descontextualizados, alejados de la referencia práctica del objeto y del contexto cotidiano y tienen que ver con la disponibilidad a tratar los procesos cognitivos como «objetos de análisis" (lo que se incluirá dentro de lo "metacognitivo»). Estas destrezas son muy necesarias en las sociedades tecnológicas, debiendo ejercitarse en contextos no sólo escolares, sino también laborales. Volveremos sobre este aspecto más adelante.

Vygotski había comprendido pronto la importancia que tiene el proceso descontextualizador que permite disponer del lenguaje como instrumento de reflexión abstracta. Para reflexionar es necesario un conjunto de significados (conceptos) desligados de la situación concreta y relativos a situaciones generales. Veíamos antes que los analfabetos uzbecos (Luria, 1979) o las vai liberianos difícilmente resolvían problemas silogísticos debido a su no aceptación de los límites del problema impuestos por las premisas. Es decir, a que no participaban de las normas convencionales de ese tipo de situaciones por las que los sujetos de las sociedades tecnológicas tienden a considerar el problema tal como se plantea, sin acudir a ningún soporte práctico. Para resolver el silogismo es necesario construir (haber construido) un conjunto de significados que sean verdaderas generalizaciones de la realidad y que, por tanto, no se soporten en contextos particulares. 
El problema del análisis semiótico del lenguaje estriba en que frente al significado, el lenguaje opone un proceso directamente ligado al contexto, el sentido, fundamental para dicho análisis. Surge así una contradicción que Vygotski asumió. Como apunta Wertsch (1985):

«El análisis semiótico de Vygotski se basa en la suposición contradictoria sobre la naturaleza del lenguaje, pues la descontextualización, después de todo, es lo contrario de la contextualización. En tanto que estas dos tendencias están en oposición, ellas operan simultáneamente para determinar la estructura e interpretación del habla. La organización de las producciones específicas puede estar dominada por una de estas fuerzas, pero la otra está casi siempre reflejada en ella."

Así pues, en ese juego dialéctico se resuelve la doble y contradictoria naturaleza del lenguaje. Y es que, como el propio Vygotski consideraba, el habla puede recorrer una amplia gama de usos psicológicos, pues igual transmite o representa una imagen particular que produce un significado muy abstracto en el desarrollo del razonamiento.

«Sólo la forma exterior del lenguaje permanece constante; su significado interno, su valor psíquico o intensidad varían libremente en función de la atención o el interés selectivo del pensamiento. Visto desde el punto de vista del lenguaje, el pensamiento se debe definir como el contenido del habla latente o potencial, el contenido que se obtiene al interpretar cada uno de los elementos en el flujo del lenguaje como poseído de su valor conceptual más elevado" (Sapir, 19, cit. por Wertsch, 1985a, p. 6).

Analizado desde esta perspectiva, el pensamiento expresado por la vía del lenguaje, en el caso de la definición de un concepto por ejemplo, representaría el máximo grado de descontextualización que este último puede expresar. En otras situaciones, nuestro habla recorrería toda esa gama de usos, si la comunicación así lo demanda.

Pero ese grado de descontextualización que caracteriza al significado en la fase final de su desarrollo ha surgido de la «interacción social psicológica», del juego entre individuos dispuestos a ponerse de acuerdo cuando la cantidad y la calidad de la información compartida lo demanda. Este sería el caso de nuestra tecnología. Dada la cantidad de información que hemos de manejar para nuestra supervivencia cotidiana, necesitamos estar de acuerdo de modo muy formalizado en nuestras representaciones, alcanzar un muy alto grado de convención social que permita la comunicación en este contexto cultural.

Esta tensión entre sentido (individual) y significado (convencional) puede romperse en ciertas situaciones experimentales, ante sujetos con otras historia cultural distinta. El hecho de que los sujetos uzbecos de Luria prefiriesen entre "hacha» y "leño" en un grupo de estímulos como "hacha", "pala" $y$ «leño» indica un predominio del sentido, que busca las relaciones de necesidad, las relaciones más altamente motivadas o sugeridas por el contexto.

\section{4. ¿HACIA UNAA PSICOLOGIA CULTURAL?}

Hasta este punto hemos presentado el panorama que de las diferencias cognitivas asociadas a la cultura ofrece la psicología transcultural. Hemos 
aceptado la idea de que no existen diferencias básicas en los procesos cognitivos de los individuos de unas culturas y otras. Más bien las diferencias que puede haber radican en el tipo de materiales y situaciones de aplicación de las destrezas y en algunas habilidades específicas de análisis de lo cognitivo. Hemos relacionado esas diferencias en el ámbito de aplicación de lo cognitivo con el tipo de actividades cotidianas propias de las sociedades tecnológicas, siendo la escuela una institución típica de ellas. Las actividades escolares recogerían muchos de los rasgos de las actividades laborales de las sociedades modernas.

Hecha una primera revisión de las aportaciones de la psicología transcultural habría que preguntarse que en qué medida podemos hablar de que ha existido una verdadera psicología cultural, capaz de recoger la intervención de los factores culturales en los procesos psicológicos. La respuesta a esta pregunta no puede ser excesivamente optimista. La casi totalidad de la investigación congnitiva transcultural ha adoptado un enfoque que a nuestro entender es erróneo. Por una parte, los investigadores se han dedicado a comparar las destrezas cognitivas de los individuos de unas y otras culturas utilizando como marco de investigación los modelos desarrollados en el laboratorio occidental y, sobre todo, utilizando como criterios de evaluación y comparación las pruebas y tareas cognitivas desarrolladas también en el laboratorio, y cuya aplicación exige su empleo, con lo que eso significa de desventaja para los no escolarizados y no occidentales. Se ha pretendido "medir a todos por el mismo rasero" de las pruebas cognitivas para ver si existían diferencias cognitivas entre los sujetos de una cultura y los de otra. El reconocimiento de que el "rasero" no eran tan igual para todos ha hecho que se intentasen modificar las condiciones de prueba, tratando de eliminar al máximo las desventajas. Pero no existe un rasero común para todos. Cualquier prueba cognitiva, cualquier situación de investigación es cultural. En otras palabras:

"No existe contexto de observación, por mucho cuidado que se hay a puesto en su construcción, que sea culturalmente neutral. Los escenarios de la conducta están organizados socialmente y se insertan en sistemas de organización mayores, que influyen sobre ellos" (Laboratory of Comparative Human Cognition, 1979, p. 829).

Pero el problema de la investigación transcultural no se agota en las tareas y las situaciones empleadas. El enfoque que ha predominado ha intentado poner en relación variables independientes que son factores culturales (escolarización, medio rural y urbano, «modernidad», etc.), definidos de modo muy genérico y difuso, con procesos cognitivos, considerados como variables dependientes (lenguaje, percepción, categorización, memoria, razonamiento, etc.), sin aclarar los mecanismos por los que esas relaciones se dan. Por una parte, la propia definición de las variables independientes es tan poco fina que se agrupan bajo un mismo rótulo (por ejemplo, escolarización) una enorme cantidad de experiencias, aprendizajes y factores asociados, cuya intervención puede ser muy diferente en relación a la variable dependiente elegida. Ha habido, por otra parte, muy pocos estudios de los mecanismos que ponen relación lo cultural y lo cognitivo. Lo que se ha hecho es una psicología de las diferencias culturales, una psicología diferen- 
cial de los individuos de las culturas. Esta psicología diferencial se ha limitado a describir diferencias sin tratar de explicar cómo llegan a generarse y cosificando a menudo lo que no eran sino sesgos de observación. En esta misma línea, Cole (1981) señala que la forma en que se ha estudiado la relación entre cultura y conocimiento ha creado una formulación de caja negra que correlaciona el input cultural y el output cognitivo, haciendo muy difícil responder a la pregunta de cómo se transforman los distintos contenidos culturales en diferencias culturales en los procesos cognitivos. En última instancia, se establecería un punto de vista dualista de la mente y de la sociedad.

La pregunta, llegados a este punto, sería entonces: ¿es posible una psicología cultural en este momento? Si las herramientas metodológicas empleadas hasta ahora, que son las de la psicología cognitiva, no sirven, en su mayoría, ¿puede desarrollarse una psicología científica que recoja la intervención de los factores culturales en el conocimiento humano? La respuesta que damos a esta pregunta es positiva. Se está empezando a elaborar en los últimos años una verdadera psicología cultural en la que aparece un enfoque de la relación entre cultura y conocimiento que se asienta en el estudio de los procesos cognitivos tal como se producen en cada cultura particular y no en la comparación de unos individuos con otros sobre la base de tareas cognitivas propias de los estudios experimentales de la psicología occidental. Este nuevo enfoque lo propugnan autores como Rogoff (1982, 1986; Rogoff y Lave, 1984), Scribner (1984), Wertsch (1984) y, especialmente, M. Cole y el "Laboratory of Comparative Human Cognition» (LCHC, 1979, 1983). Este nuevo enfoque surge en el marco teórico de la psicología sociocultural desarrollada por Vygotski y sus seguidores (Vygotski, 1977, 1979; Luria, 1979; Wertsch, 1985b).

La idea fundamental del enfoque vigotskiano es el carácter social, y por tanto histórico, de los procesos cognitivos. El funcionamiento cognitivo emergería de la interacción social. Para Vygotski toda función psicológica individual ha sido antes una función interpsicológica, social. En sus propias palabras:

«Cualquier función del desarrollo cultural del niño aparece dos veces o en dos planos. Primero aparece en el plano social y después en el plano psicológico. Primero aparece entre personas como una categoría interpsicológica y después dentro del niño individual como una categoría intrapsicológica» (Vigotsky, 1979).

La conversión de lo interpsicológico en intrapsicológico sería el resultado de un proceso de internalización que, para Vigotsky, es «la reconstrucción interna de una operación externa». Este proceso conllevaría unas transformaciones:

1. Una operación que inicialmente es externa se reconstruye y comienza a ocurrir internamente.

2. Un proceso interpersonal se transforma en otro intrapersonal. Toda función se da primero en el plano social, para darse por fin en el plano individual.

3. La transformación de un proceso interpersonal en un proceso intrapersonal es el resultado de una larga evolución. Por otra parte, la inter- 
nalización iría unida a cambios en las leyes que rigen la actividad y se incorpora en un nuevo sistema con sus propias leyes. La actividad psicológica internalizada se reconstruiría en un nuevo plano.

Junto a la idea del origen social de los procesos psicológicos hay otras en este enfoque de gran interés para la psicología cultural: el concepto de actividad y la noción de zona de desarrollo próximo.

El concepto de actividad ocupa un lugar central en la psicologia soviética. A. N. Leontiev (1973-74, 1981) fue quien lo formuló con más claridad. Este concepto proporciona la unidad de análisis de lo psicológico. Como una unidad de análisis de lo psicológico, la actividad sería un concepto que supera el esquema E-R, permitiendo considerar alos procesos empleados por los sujetos activos para formar conexiones reales con el mundo de los objetos» (Leontiev, 1973-74, cit. por Cole, 1981).

El interés de la idea de actividad para la psicología cultural reside en que convierte en objeto de estudio de la psicología la actuación de los individuos concretos, tal como tiene lugar en el seno de la sociedad. Dice Leontiev:

"Si separamos la actividad humana del sistema de relaciones sociales y de la vida social, no existiría... La actividad humana individual es un sistema dentro del sistema de relaciones sociales. No existe sin estas relaciones" (Leontiev, 1973-74).

La importancia del concepto de Leontiev viene dada porque en él se condensan tanto los planos intelectuales (planificación) y prácticos de la conducta, como la dimensión social que esta tiene (acciones compartidas, por ejemplo).

La noción de zona de desarrollo próximo se debe a Vygotski (1979). Se define como «la distancia entre el nivel de desarrollo actual del niño, determinado por la resolución independiente de problemas y el nivel de desarrollo potencial, determinado por la resolución de problemas bajo la guía del adulto o en colaboración con compañeros más capaces» (Vygotski, 1979, p. 133).

La noción de zona de desarrollo próximo es de gran interés para el estudio de las relaciones entre cultura y conocimiento. Para Cole (1981) y el LCHC (1983), este concepto tiene una importancia clave como punto de encuentro de lo cultural y lo cognitivo («donde cultura y conocimiento se generan mutuamente»). Los contextos que organizan la transformación de lo social a lo psicológico del pensamiento serían «zonas de desarrollo próximo" (ZDP) donde el sujeto va realizando una parte muy importante de sus aprendizajes $y$, sobre todo, los de aquellas destrezas que tienen un carácter claramente cultural.

En la psicología actual y en la antropología pueden encontrarse estudios en los que aparecen descritas formas de actividad compartida en la que se observa cómo ciertas destrezas culturales devienen destrezas cognitivas a través de la internalización en la ZDP. Buenos ejemplos de esto serían los trabajos de Wertsch y cols. (Wertsch, 1979, 1985c; Wertsch, Minick y Arns, 1984) o las aportaciones de Rogoff (Rogoff, 1986; Rogoff y Gardner, 1984).

Podemos reseñar dos investigaciones que nos dan una visión de los ob- 
jetivos que la psicología cultural debe cubrir. Wertsch, Minick y Arns (1984) han observado diferentes pautas de regulación extrapsicológica en situaciones de interacción adulto-niño. Específicamente observaban y comparaban la forma en que resolvían un problema unas parejas compuestas por madres brasileñas de bajo nivel sociocultural y sus hijos de seis años y parejas compuestas por profesoras de la misma comunidad y sus alumnos de seis años igualmente. Las diferentes pautas de regulación que se observaron (más directamente orientada hacia la ejecución de la tarea en las madres; más encaminada hacia la asunción de responsabilidad por parte del niño en las profesoras) produjeron diferencias en el grado de autocontrol (regulación intrapsicológica) que unos y otros niños alcanzaban. Más específicamente, las profesoras tendían a desarrollar una forma «indirecta» de regulación sobre el niño que se plasmaba en formas abreviadas de comunicación, preguntas y, en general, el empleo de recursos semióticos que movilizaban al niño hacia una mayor iniciativa y autonomía. Las madres, por el contrario, eran más directivas, propensas a emitir expresiones largas, casi sin abreviación, que tendían a reducir la autonomía del niño.

En otro trabajo de Childs y Greenfield (1982) se estudiaba el modo en que aprenden a tejer las muchachas del grupo zinancateco de Chiapas (México). La primera parte de la investigación consistía en un cuidadoso estudio del papel de la dirección social en el dominio de la actividad de tejer. Esta dirección adoptaba dos formas. En la primera de ellas, la tejedora intervenía en los momentos de la tarea en que las aprendizas podían cometer faltas. La intervención adulta era también mayor al comienzo de cada paso de la actividad que al final de éstos. En la instrucción adulta era importante el papel del lenguaje. El habla de las tejedoras estaba muy relacionada con el grado de destreza de cada niña y con las circunstancias específicas con las que tenía que enfrentarse. Así, en los primeros momentos del aprendizaje predominaban las órdenes del siguiente tipo: «Haz...», referidas a una actividad general. Eran órdenes que describían el conjunto de la tarea. Posteriormente el habla adulta iba cambiando para señalar los momentos más críticos de la actividad, o para establecer vínculos entre diferentes fases de dicha actividad.

La segunda forma en que las tejedoras zinancatecas dirigían la práctica de las aprendizas era haciendo que éstas estuviesen implicadas en todos los pasos del proceso de tejido y asociasen las actividades como parte del aprendizaje de cada uno de los pasos.

Es dentro del marco teórico del enfoque sociocultural soviético donde se está desarrollando una psicología que recoge las relaciones entre cultura y conocimiento. Coincidimos con este enfoque en que lo cultural y lo cognitivo no son sino dos caras de un mismo fenómeno, en que existe una unidad indisociable entre ambos aspectos. Como afirma $M$. Cole citando a Vygotski:

«... el individuo y lo social se concebían como elementos mutuamente constituyentes de un sistema interactivo único: se contemplaba el desarrollo cognitivo como un proceso para adquirir la cultura» (Cole, 1981, p. 5 de la traducción castellana).

¿Cómo debe, pues, proceder la investigación en psicología cultural? Desde una perspectiva heredera del enfoque sociocultural, recogiendo, ade- 
más, las aportaciones de la etnometodología, puede decirse que la investigación debe comenzar por un análisis detallado de las actividades cotidianas que realizan los individuos en su relación con el ambiente cultural para, una vez conocidas esas actividades cotidianas, determinar cuáles son los rasgos y destrezas cognitivas que dichas actividades conllevan y exigen. El análisis no se centra en las personas individuales ni en las dimensiones culturales abstractas, sino en los contextos culturales (LCHC, 1983) y, más especificamente, en las actividades desarrolladas en dichos contextos culturales. Estos contextos serían los escenarios fundamentales en los que se construye el desarrollo cognitivo. Son situaciones socialmente ensambladas para la acción y la resolución de problemas, construidas por los individuos en interacción con otros. Las prácticas culturales que tienen lugar en esas situaciones conectadas socialmente «han de aprenderse como sistemas de actividad. Estos escenarios tiene reglas permanentes, lo que los psicólogos cognitivos denominan "guiones" (scripts), los antropólogos "contextos" y los sociólogos "expectativas de fondo", que orientan a las personas hacia la conducta que es apropiada para una situación determinada.» (LCHC, 1983, p. 333.)

Los contextos culturales no pueden igualarse al ambiente físico; éste forma parte del contenido cultural donde se produce la conducta. Son construidos por los seres humanos, por lo que las personas están haciendọ, cuándo lo hacen y cómo lo hacen. Es decir, los seres humanos sirven como «ambientes» para los demás.

Desde este punto de vista, las prácticas culturales se aprenden mediante «esquemas» que ponen en relación directamente experiencias culturales y ejecución de destrezas cognitivas. Este concepto de esquema tiene mucho que ver con el de actividad citado anteriormente, ya que ambos establecen continuidad entre la experiencia cultural y la destreza cognitiva como elementos de una misma unidad. Las experiencias culturales incluidas en estos segmentos de análisis son situaciones de interacción social en las que se aprenden destrezas culturales-cognitivas; son contextos de regulación (zonas de desarrollo próximo) donde se produce la transmisión de destrezas que son, de modo indisociable, culturales y cognitivas. Es aquí donde la noción de zona de desarrollo próximo nos permite caracterizar las situaciones de transmisión social de destrezas. Los mecanismos de influencia de los agentes socializadores sobre los niños son variados. Podemos presentar algunos de ellos (LCHC, 1983):

1. La cultura ordena cuándo se presentan ciertos ambientes específicos de resolución de problemas, encarnados en las prácticas culturales.

2. La frecuencia de la práctica básica está culturalmente organizada.

3. La cultura modela las pautas de ocurrencia simultánea de los acontecimientos.

4. La cultura regula el nivel de dificultad de la tarea dentro de los contextos.

Se puede hacer ya una caracterización completa de cómo debe proceder la investigación en psicología cultural. En primer lugar, es necesario analizar las actividades cotidianas que llevan a cabo los individuos, y que son de signo cultural, es decir, las prácticas culturales. Interesan muchos tipos de actividades: 
- Las relacionadas con la producción material, es decir, las actividades laborales.

- Las experiencias educativas de interacción dentro de la familia.

- Las experiencias educativas de indole formal e informal características de la cultura fuera del marco familiar (la escuela, si existe, el aprendizaje de oficios y otras actividades relevantes).

Las nociones de actividad y zona de desarrollo próximo son dos buenas herramientas conceptuales. La de actividad permite el análisis de lo que los individuos socialmente organizados hacen cotidianamente y cuál es la dimensión cognitiva de dicha actuación. La noción de ZDP es útil para la tarea de determinar las situaciones de interacción social en que se adquieren las destrezas culturales o, lo que es lo mismo, la internalización de las acciones que las constituyen.

Tras este análisis, tiene sentido el empleo de la tecnología experimental (con las tareas propias de la cultura) como instrumento para determinar de forma rigurosa las destrezas cognitivas socialmente adquiridas. La experimentación debe hacerse teniendo en cuenta las características del contexto cultural, empleando variables extraídas de la misma realidad en estudio. De esta manera se reduciria el apriorismo que ha caracterizado a muchas investigaciones transculturales.

\section{Referencias}

BERLIN, B. (1972): «Speculation on the growth of ethnobiological nomenclature.* Journal of Language and Society, 1, 63-98.

BerliN, B. (1978): «Ethnobiological classification». En E. Rosch y B. B. Lloyd (eds.): Cognition and categorization. Hillsdale, N. J.: L. E. A.

BroWN, A. L. (1977): *Development, schooling and the acquisition of knowledge about knowledge». En R. C. ANDERSON; R. J. SPIRO y W. E. MONTAGUE (eds.): Schooling and the acquisition of knowledge. Hillsdale, N. J.: L. E. A.

BRUNER, J. R.; GREENFIELD, P. M., y OLVER, R. (1965): Studies in cognitive growth. N. York: Wiley.

Childs, C. P., y GReEnfield, P. M. (1982): «Informal modes of learning and teaching: The case of Zinacanteco weaving». En N. WARREN (ed.): Studies in cross-cultural psychology, Vol. 2, N. York: Academic Press.

COLE, M. (1984): *The Zone of Proximal Development: Where culture and cognition create each other." Separata del Center for Human Information Processing, 1981 (versión castell. en Infancia y Aprendizaje, 25, 3-17).

COLE, M.; GAY, J. y GLICK, J. (1969): "Communications skills among the kpelle of Libreria». Articulo presentado a la Society for Research in Development Meeting, Santa Mónica, California, marzo.

COLE, M.; GAY, J.; GLICK, J. y SHARP, D. W. (1971): The cultural context of learning and thinking. N. York: Basic Books.

COLE, M., y SCRIBNER, S. (1974): Culture and thought: A psychological introduction. N. York: Wiley. (Traducc. castell. en México: Limusa, 1977.)

COLE, M., y SCRIBNER, S. (1977): „Cross-cultural studies of memory and cognition*. En R. V. KaIl y J. W. FHAGEN (eds.): Perspectives on the development of memory and cognition. Hillsdale, N. J.: L. E. A.

DEREGOWSKI, J. B. (1970): «Effect of cultural value of time upon recall». British Jourmal of Social and Clinical Psychology, 9, 37-41.

DeRegowsKI, J. S. y SERPELL, R. (1971): «Performance on a sorting task with various modes of representation: a cross-cultural experiment». Human Development Research Unit. University of Zambia. Informe $n .^{\circ} 18$.

DUBE, E. F. (1982): *Literacy, cultural familiarity, and "intelligence" as determinants of story recall.. En U. NEISSER (ed.): Memory observed. Remembering in natural contexts. San Francisco: W. H. Freeman.

FJELLMAN, A. S. (1971): The mith of primitive mentality: $A$ study of semantic acquisition and 
modes of categorization in Akamba children of South Central Kenya. Tesis doctoral inédita. Universidad de Stanford.

GAY, J. (1971): «Kpelle uses of kpelle logic*. Liberian Research Association Journal, 4.

GoODNOw, J. J. (1976): *The nature of intelligent behavior: Questions raised by cross-cultural studies". En L. B. ResNick (ed.): The nature of intelligence. Hillsdale, N. J.: L. E. A.

GREENFIELD, P. M. (1974): "Comparing dimensional categorization in natural and artificial contexts: A development study among the Zinacantecos of Mexico». Journal of Social Psychology, 93, 157-171.

GREENFIELD, P. M. y LAVE, J. (1982): "Cognitive aspects of informal education". En D. A. WAGNER y H. W. STEVENSON (eds.): Cultural perspectives on child development. San Francisco: W. H. Freeman.

IRWIN, M. H., y McLAYGHLIN, D. H. (1970): «Ability and preference in categorization sorting of Mano school children and adults». Journal of Social Psychology, 82, 15-24.

IRwIN, M. H.; SCHAFER, G. N., y FEIDEN, C. P. (1974): «Emic and unfamiliar category sorting of Mano farmers and U.S. undergraduatesn. Journal of Cross-Cultural Psychology, S, 407-423.

LABORATORY of COMPARATIVE HUMAN COGNITION (1979): «Cross-cultural psychology's challenges to our ideas of children and development w. American Psychologist, 34, 827-833.

LABORATORY of COMPARATIVE HUMAN COGNITION (1983): «Culture and cognitive development". En W. KESSEN (ed.): Handbook of child psycology: History, theory and methods (Vol. 1). N. York: Wiley.

Leontiev, A. N. (1973-74): «The problem of activity in psychology». Soviet Psychology, 13, 4-33.

LEONTIEV, A. N. (1981): El desarrollo del psiquismo. Barcelona: Akal.

LURIA, A. R. (1979): Los procesos cognitivos. Análisis socio-bistórico. Barcelona: Fontanella.

MANDleR, J. M.; SCRIBNER, S.; COlE, M., y DeFOREST, M. (1980): “Cross-cultural invariance of story recall.» Child Development, S1, 19-26.

OLSON, D. (1977): "The languages of instruction: On the literate bias of schooling». En R. C. AANDERSON; R. J. SPIRO y W. E. MONTAGUE (eds.): Schooling and the acquisition of knowledge. Hillsdale, N. J.: L. E. A.

PARIS, S. G.; NEWMAN, D. R., y JACOBS, J. E. (1985): «Social contexts and functions of children's rememberingw. En C. J. BRAINERD y M. PRESSLEY (eds.): Cognitive learning and memory in children. N. York: Springer-Verlag, 1985.

PICK, A. D. (1980): "Cognition: psychological perspectives". En H. C. Triandis y W. J. LONNER (eds.): Handbook of cross-cultural psychology (Vol. 3). Boston: Allyn \& Bacon.

PrICE-WILliams, R. D. (1962): «Abstract and concrete modes of classification in a primitive society.* British Journal of Educational Psychology, 32, 50-61.

RAMÍREZ, J. D.; CASADO, F.; GASCON, M.; MARTínez, R., y de la MATA, M. (1986): Influencia de la cultura en el aprendizaje y el pensamiento: una investigación en torno al procesamiento semántico en adultos analfabetos. Memoria de investigación.

ROGOFF, B. (1981): «Schooling and the development of cognitive skills». En H. C. TRIANDIS y A. Heron (Eds.): Handbook of cross-cultural psychology (Vol. 4). Boston: Allyn \& Bacon.

ROGOFF, B. (1982): *Integrating context and cognitive development». En M. E. LAMB y A. L. Brown (eds.): Advances in Developmental Psychology (Vol. 2). Hillsdale, N. J.: L. E. A.

ROGOFF, B. (1986): «Adult assistance of children's learning». En T. E. RAPHAEL (Ed.): The contexts of school-based literacy. N. York: Randon House.

ROGOFF, B., y GARDNER, W. (1984): «Adult guidance of cognitive development". En B. RoGOFF y J. LAVE (Eds.), op. cit.

ROGOFF, B., y LAVE, J. (eds.) (1984): Everyday cognition: its development in social context. Cambridge, Mass.: Harvard University Press.

RogofF, B., y MISTRY, J. (1985): «Memory development in cultural context . En M. PRESSLEY Y C. J. BRAINED (eds.): Cognitive learning and memory in children. N. York: Springer-Verlag.

ROGOFF, B., y WADDEL, K. J. (1982): «Memory for information organized in a scene by children from two cultures». Child Developmen, 53, 1224-1228.

RosCH, E. (1975): «Cognitive representations of semantic categories». Joumal of Experimental Psychology: General, 104, 3, 192-233.

SCRIBNER, S. (1974): $*$ Developmental aspects of categorized recall in West Africa». Cognitive Psychology, 6, 475-494.

SCRIBNER, S. (1977): *Modes of thinking and ways of speaking*. En P. N. JOHNSON-LAIRD y P. C. WASON (1ds.): Thinking: readings in cognitive science. N. York: Cambridge University Press.

SCRIBNER, S. (1984): «Studying working intelligence». En B. ROGOFF y J. LAVE (eds.), op. cit.

SCRIBNER, S., y COLE, M. (1973): *Cognitive consequences of formal and informal education». Science, 182, 553-559 (trad. castell. en Infancia y Aprendizaje, 1982, 17, 3-18). 
SCRIBNER, S. y COLE, M. (1981): The psychology of literacy. Cambridge, Mass.: Harvard University Press.

SERPELL, R. (1969): «Cultural differences in attention preference for color over form*. International Journal of Psychology, 4, 1-8.

SHARP, D.; COLE, M., y LAVE, J. (1979): «Education and cognitive development: The evidence from experimental research. Monographs of the Society for Research in Child Development, 44 (1-2 Serial $\left.n .^{\circ} 178\right)$.

Soтo, P. (1981): Adquisición de términos y formación de conceptos. Un estudio evolutivo. Tesis Doctoral inédita. Universidad Complutense de Madrid.

Stevenson, H.W. (1982): *Influences of schooling on cognitive development». En D. A. WAGNER y H. W. STEVENSON (eds.): Cultural perspectives on child development. San Francisco: Freeman.

VyGOTSKI, L. S. (1977): Pensamiento y lenguaje. Buenos Aires: La Pléyade.

VYGOTSKI, L. S. (1979): El desarrollo de los procesos psicológicos superiores. Barcelona: Crítica.

WAGNER, D. A. (1978): «Memories of Marrocco: the influence of age, schooling and environment on memory». Cognitive Psychology, 10, 1-28.

WAGNER, D. A. (1981): «Culture and memory development». En H. C. TRIANDIS y A. HERON (eds.): Handbook of cross-cultural psychology (Vol. 4). Boston: Allyn \& Bacon.

WERTSCH, J. V. (1979): «From social interaction to higher psychological processes: A clarification and application of Vygotsky's theory». Human Development, 22, 1-22.

WERTSCH, J. V. (1984): "The zone of proximal development: some conceptual issues». En B. ROGOFF y J. V. WERTSCH (eds.): Children's learning in the zone of proximal development, n. ${ }^{\circ} 23$ New Directions for Child Development. San Francisco: Jossey-Bass.

WERTSCH, J. V. (1985a): *The semiotic of mental life: L. S. Vygotsky and M. M. Bakhtin». En E. MerTz y R. J. PARMENTIER (eds.): Semiotic mediation: Psychological and sociocultural perspectives. N. York: Academic Press.

WERTSCH, J. V. (1985b): Vygotsky and the social formation of mind. N. York: Harvard University Press.

WERTSCH, J. V. (1985c): aAdult-child interaction as a source of self-regulation in children". En S. YUSSEN (ed.): The growth of insight during childhood. N. York: Academic Press.

WERTSCH, J. V.; MINICK, N., y ARNS, F. J. (1984): «The creation of context in joint problem-solving». En B. ROGOFF y J. LAVE (eds.).

Wilkinson, A.; PARKer, T., y STEVENSON, H. W. (1979): «Influence of school and environ-

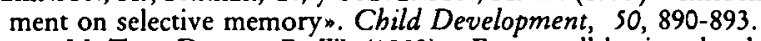

ZIVIAN, M. T., y DARJES, R. W. (1983): «Free recall by in-school and out-of-school adults: performance and metamemory $*$. Developmental Psychology, 19, 513-520.

\section{Extended summary}

During the last decades, cognitive cross-cultural psychology has established a number of differences between Western and non-Western subjects. The aim of this paper is to review these differences and the research in this area, in order to examine their contributions to the development of a genuine cultural psychology.

Studies on cognitive processes like perception, classification, memory an reasoning have showed that, in general, non-Western subjects (specially those lacking school experience) ussually think on the basis of contextbound features of the stimuli rather than on the their logical and abstract characteristics.

However, a more detailed analysis of cross-cultural investigations shows that there are a great number of methodological problems that question the validity of the data they provide. Issues like familiarity with material or with test situations, specially those related to the objective of activity are at the basis of the differences. The existence of social settings, in wich the need to use cognitive processes is not clearly defined by the practical requirements of the situation (for instance, in schools or psychological tests) is characteristic of Western societies and not very common in non-Western cultures. 
The differences between Western and non-Western subjects lies in the situations where cognitive processes are applied. At the same time, there is an important difference linked to the verbal mediation of activity. NonWestern subjects rarely verbalize the criteria they use. Following the Vygotskian line of argument, it can be said that thinking in modern cultural settings is supported on the decontextualizer potentiality of speech. This makes possible what cognitive psychologists term metacognition.

The emergence of a genuine cultural psychology, starts from the recognition that it is not possible to design contexts for observing cognitive processes that can be free of cultural influence. In order to overcome this problem it is necessary to adopt an approach which goes beyond a black-box perspective and lets us specify the complex mechanisms by which cultural experiences shape cognitive processes. Concepts like activity and zone of proximal development, proposed by Vygotsky and other Soviet theorist offer the possibility of integrating and studying the cognitive-cultural determinants of human development. Authors like Wertsch, Rogoff and others, are currently developing research aimed at disentangling the mechanisms by which cultural activities model cognitive skills. We review two examples of investigation which adopt this perspective.

Starting from a sociocultural approach, psychological investigation should begin by a detailed analysis of everyday activities that individuals carry out in their relation to cultural settings. This kind of analysis must permit us to determine the cognitive skills and features involved in cultural practices. Activities like the following have a special interest for our purpose:

- Socio-working activities

- Educational activities in the family family.

- Formal and informal educational activities taking place out side the

The notions of activity and zone of proximal development can be two useful conceptual tools for analysing the processes by which "culture and cognition create each other.»

After the preceding analysis, the employ of experimental methodology can serve us to determine, in a more precise way, how cognitive skills are culturally acquired. 